\title{
Enriching Quality of Maternal Health Care through Machine Learning
}

\author{
Bolanle F. Oladejo \\ Computer Science Depatment \\ University of Ibadan, \\ Ibadan, Oyo State, Nigeria
}

\author{
Oladejo Olajide A. \\ Computer Science Department \\ University of Ibadan, \\ Ibadan, Oyo State, Nigeria
}

\begin{abstract}
Pregnancy outcomes rank the most pressing reproductive health problems in the world globally. Most maternal complications and deaths occur as a result of insufficient quality of care during pregnancy and labour. In most parts of the developing world, access to quality health care is limited and people depend on the health care providers who have limited training. Advancements in medical technology have drastically increased the quantity of data available in the healthcare industry, ranging from patient reports and genomic data to electronic medical records. These data can provide a wide scope of insights into patient cases for prevention and cure on health issues. Thus, this research aimed at designing a machine learning-based decision support system for maternal health care. The system is designed using Unified Modeling Language (UML) tools and a multi-class Support Vector Machine (SVM) was developed for the Decision Support System for Maternity Health Care (DSSMC). A Web-based DSSMC was developed and tested to facilitate automatic diagnosis of patient and to solve the problem of human error and bias.
\end{abstract}

\section{General Terms}

Knowledge Management, Health Care, Machine, Support Vector Machine

\section{Keywords}

Maternal Health, Diagnosis, Decision Support System, Machine Learning, Knowledge Management.

\section{INTRODUCTION}

In the global world, pregnancy ranks among the most pressing reproductive health problems and statistics has shown that an annual estimate of 600,000 women aged $15-49$ died of pregnancy-related causes, with 99 per cent coming from the developing world $[16,252,22,17]$ and Nigeria alone accounting for over 10 per cent of the total figures [14]. The World Bank (1998) had realized that maternal and infant mortality depends to a large extent on whether women have access to information, education and communication resources required to provide themselves and their infants with adequate care. Most maternal complications and deaths occur as a result of insufficient quality of care during pregnancy and labour. In much of the developing world, access to quality health care is limited and people depend on the health care providers who have limited training or supervision. Further evidence suggests that overburden health workers are overwhelmed and almost one-fourth of time of health care workers was spent in non-clinical work (including documentation), it is possible that the burdened workforce may have an impact on the quality of delivery of health services.
A decision support system may be any system which helps decision-makers to make decisions in the health care system. It can be any computer program designed to help health professionals to make clinical decisions. It is defined as an active knowledge-based system (KBS) that uses items of patient data to generate case-specific advice [20]. This system can help the user make the best decision in terms of the combination of mortality and complications. Both machine learning and optimization technique are utilized in this decision support system. This system helps in making diagnostic decisions in a sequential decision-making environment by identifying the most promising tests and suggesting a diagnosis, this can improve the diagnostic process, reduce consumption of medical tests, save costs, and give an efficient diagnosis. Another important element in the clinical decision support system architecture is an electronic medical record (EMR). EMR in this system acts as the input of the inference engine and smooth's the process of entering patient information. EMR has been widely implemented in most of the hospital information system nowadays for its benefits in health care [11], and such systems have not been seen in Nigeria. Study had also shown that EMR can improve the quality of care when integrated with a clinical decision support system [3]. Therefore, electronic medical record is selected to be included in the Clinical Decision Support System (CDSS) architecture. The main function of this component is to facilitate the process of retrieving patient data. Advancements in medical technology have drastically increased the quantity of data available in the healthcare industry, ranging from patient reports and genomic data to electronic medical records and more. These data can provide a wide scope of insights into patient cases for prevention and cure. Predictive analysis leverage these large, heterogeneous data sets to further knowledge and foster discovery which can identify similarities among patients based on a wider scope of variables or characteristics such as age, genetic marks, previous illness, etc., leading to finer groupings and thus more accurate predictive analysis. Such kind of predictive method can facilitate appropriate and timely care by forecasting an individual's health risk, clinical course, or outcome, uncovering these types of patterns at an early stage can be of great benefit to both the doctors and patients and can explain the use of drug combinations and treatments independent of any promotion by mapping the predicted targets, compared to statistical methods, machine learning can increase prediction accuracy with fewer assumptions. Figure 1. Shows a schematic diagram of a Clinical Decision Support System (CDSS). 


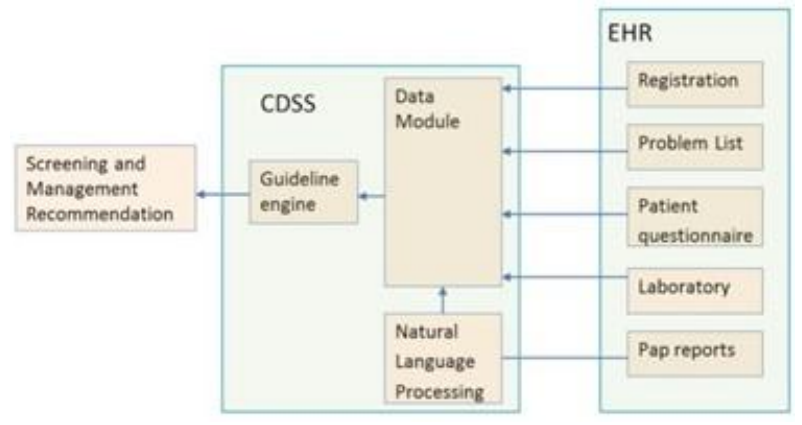

Fig 1: Schematic diagram of a Clinical Decision Support System for Electronic Health Record [23].

Decision Support System (DSS) stores and use knowledge when information is requested. The knowledge of this decision support system tools usually comes from the direct input from human experts. The proposed system gathers knowledge automatically and uses an optimization method to return appropriate answers to requested information's (query). In this project the use of electronic health record with data mining techniques to manage patient's information, to facilitate personalized health care, to improve outcomes and reduce the risk of complications and maternal issues. Using different records overtime after taking the qualitative and quantitative data like the history of the patients over trimesters, can be used to project the likely symptom and diagnosis of an antenatal patient when there is a report with the help of a machine-learning algorithm to make predictions which will aid timely decision and proper address of similar issues and help in control the increase in the rate of maternal complications and infant mortality. To achieve the goal of diagnosis, a good balance of diagnostic accuracy and duration of diagnosis. In this paper, we propose a system using UML tools and a multi-class Support Vector Machine SVM for the Decision Support System for Maternal Health Care (DSSMC).

The organization of this paper is as follows. Section (2) consists of the theoretical background and related works. In (3), we discussed the materials and methods used in developing the DSSMC system. In Section (4), the research findings were highlighted and we summarize the paper in Section (5).

\section{RELATED WORKS}

Identifying and managing the risks associated with pregnancy is essential to providing appropriate treatment to pregnant women (National Institute for Health Care Excellence, 2008). With the use of a clinical decision support system for making a timely decision, it has been seen that the time it takes to identify and manage the risk associated with pregnancy is been controlled. The diagnosis of an illness can be done in various ways; from the patient's description, physical examination and/or laboratory tests. After the completion of diagnosis by an expert or a doctor, treatment is given keeping in mind drug reactions and allergies. However, in some cases, there is a chance of wrong diagnosis due to human error which may lead to drug reactions and allergies in a patient and may lead to life-threatening situations. To address these points, the Clinical Decision Support System helps healthcare professionals in problem-solving during medical diagnosis. [9] observed that Clinical Decision Support Systems (CDSSs) appreciably improved clinical exercise in $68 \%$ of trials and five system features were significantly more likely to enhance clinical practice than interventions lacking the feature. In 2017, a group of researchers were able to predict pregnancy outcomes among Systemic lupus erythematosus affected women with the help of a Clinical Decision Support System (CDSS), a condition which is highly associated with poor obstetric outcomes. Based on the values for 16 factors the CDSS predicts the outcome of the pregnancy as either spontaneous abortion or live birth [15]. [6] found that CDSSs improved practitioner performance in $64 \%$ of the studies, the result includes that 4 of 10 diagnostic systems, 16 of 21 reminder systems, 23 of 37 disease control systems, and 19 of 29 drug dosing or prescribing systems. However, only $13 \%$ of studies improved patient outcomes.

[1] Designed a support system to improve medical resource allocation of Bejaia region. The study collects information about patients from January 2015 through December 2015 from various Bejaia department health sector institutions. Data integration was performed in a data warehouse using the multi-dimensional model and OLAP cube and made use of Microsoft SQL Server 2012 and Microsoft Excel 2010 during implementation. The outcome involves the development of a decision support platform and was implemented during the planning stages allowing the management of different medical orientations, it provides better appointment and allotment of medical resources and ensures that the distribution of health care resources has optimal effects on improving health. It was concluded that a decision support system was designed and implemented to improve health care in Bejaia department to especially assist in the selection of the optimum location of health centre and hospital, the speciality of the health centre, the medical equipment and the medical staff.

[8] Undertake a survey of the empirical evidence on computerised decision support systems, their contexts of use, and to summarize the effectiveness of the tools and insights into how they can be successfully implemented and adopted. A systematic literature reviews on computerised decision support systems and their impact on the quality and safety of healthcare delivery over 13-year period (1997-2010) was carried out to achieve this. Various kinds of databases were searched which include The Cochrane Database of Systematic Reviews, Database of Abstracts of Reviews of Effects, The Cochrane Central Register of Controlled Trials etc. For the research to be eligible for inclusion, systematic reviews needed to address computerised decision support systems and at least one of the following; impact on safety, quality, or organisational implementation or adoption considerations. At the end of the review, the searches yielded 121 systematic reviews relating to eHealth, 41 was identified as investigating computerised decision support systems. This indicated that there was a lack of investigating potential risks, such system can result in improvements in practitioner performance in the promotion of preventive care and guideline adherence. However, the evidence concerning the impact on patient outcomes was less clear-cut with reviews finding either no, inconsistent or modest benefits. It was finalized that, whilst the potential of CDSS is improving, practitioner performance is substantial, such innovation may likewise introduce new risks resulting not only from the technical challenges (such as data inaccuracies) but also from disruption of clinical workflows.

\subsection{Knowledge Management in Health Care}

Knowledge management has become very important in the healthcare sector as a medical practice requires tools to extend the mind's limited capacity to recall and process large numbers of relevant variables. There is no assurance that professionals will learn new knowledge relevant to their patient's problem as most of their knowledge as become 
obsolete. Indeed, even with inaccessibility of knowledge to retain, a doctor's self-sufficient mind cannot reliably incorporate that knowledge with the infinite variety of data about patients to identify and systematically assess all diagnostic or treatment options based on each patient's unique characteristics and needs [24. The need for knowledge management tools has been more widely recognised and healthcare providers have chosen knowledge management approach that suits their needs as the amount of healthcare knowledge keeps growing. Many healthcare organisations exploit a reuse model, but some organisations conduct the personalisation strategy. The codification strategy is emphasised because the medical information is fundamentally the same from doctor to doctor. Though doctors may specialize in different areas, but the treatment given to patients for certain illness remains the same, therefore, clinical guidelines can be codified and the information can be reused across the nation. Also, administrative documents can be stored in the database where they can be retrieved and updated easily when needed by doctors. By so doing, knowledge management tools address the first stage decision making and support information retrieval which is used to improve the capacity of decision making [7]. The decisions of patient's care should be based on best available knowledge applied in line with point-of-care patient data and compliance with the patient's therapeutic preferences. The present research shows that the failure of physicians to access and apply relevant knowledge healthcare leads to the delivery of suboptimal care to patients [13].

While knowledge management systems use information technologies to manage the creation, storage, sharing, and use/reuse of knowledge; health care presents a special challenge to the use of knowledge management such as the complexity of the system, effect of medical errors, substantial growth of knowledge in the medical field, and an increased health care cost. Knowledge management is able to assist in medical errors reduction, and consequently their cost by providing decision support for practitioners by using either case-based reasoning and/or rule-based reasoning. Studies showed that lack of cooperation in health care is a leading cause of many medical mistakes, hence the need for coordinated inter-professional strategy. Thus, cooperative diagnosis can be achieved by the health care actors via the implementation of knowledge management systems and therefore, enhancing the quality of care by finding, sharing, collaborating and developing clinicians of knowledge to discover and develop knowledge [5].

As reported in [10], investigation was carried out on knowledge management implementation and knowledge management tools used in healthcare for informed decision making and found that utilization of knowledge management in healthcare is improving, there are numbers of opportunities for knowledge management implementation, though there are some barriers as well. Some kinds of opportunities that can transform healthcare are advances in health information and communication technology, clinical decision support systems, electronic health record systems, communities of practice and advanced care planning. It was concluded that providing the right knowledge at the right time at the point of decision making by implementing knowledge management in healthcare is paramount and to do so, one has to use the appropriate tools for knowledge management and userfriendly system because it can significantly improve the quality and safety of care provided for patients both at clinic and home settings. A systematic knowledge management approach of patient's healthcare to facilitate access to reliable, relevant medical information with adequate depth was suggested by [19] and it was concluded that the clinical knowledge base of patient's healthcare is a constructive example of the company's successful knowledge management.

An existing generic knowledge management systems model was formulated in [12] by extending an organisational and system factors relevant to healthcare, the model was tested by using a New Zealand data obtained from 263 doctors working with two district health boards and it resulted in knowledge content quality which was found to be particularly important for knowledge management systems success with respect to the system factors and of the organisational factors, leadership was the most important and more important than incentives. It was concluded that leadership promoted knowledge management systems success primarily by positively affecting knowledge content quality.

\section{MATERIALS AND METHODS}

The focus of this work is to provide the health care providers a support system which will aid in making timely decision whenever there are pregnancy-related complaints and also makes it easier for them to retrieve and access patient information/medical history from the repository which will be made available. The following sections analyse the technique and approach used in implementing the decision support system for maternity health care.

\subsection{Data Source}

The traditional relational database has been the significant data source. The other major source of data is machinegenerated (Synthetic) and real-time data, such as from the internet of things (IoT) devices. Websites o social media are scraped with data mining tools to generate data, data are also generated by machine in the form of transactions and log files. Human interactions produce data in the form of text and email messages, images, and videos over digital media. The human brain is versed at extracting information from these varied media formats. In disparity, this kind of data is challenging for computers to understand. Machines tend to produce structured data, while humans produce unstructured data [21]. A data source is a source of data which can either be a file, a database or a live data feed. The data might be located on the same computer as the program or another computer which is on the network. The main purpose of data source is to gather the technical information needed to access data into a single place and ensure it is hidden from the user without knowing where the data resides and how applications get it.

\subsection{Architecture of DSSMC}

The patient is being registered by an admin in the medical centre and a patient file is being created in the repository, the patient details are sent to a nurse to take the necessary vital signs which is thereafter assigned to a doctor for further consultation. The doctor takes down the patient's complaints/symptoms and patient is sent to the laboratory for some test to be carried out, the complaints are then sent to the decision support system where the actual diagnosis and treatment is being gotten from by using a classification method with support vector machine in modelling the data for prediction of likely complications that the system has been trained with from the past. After the whole consultation is done the doctor then generate a report based on the consultation case and the patient case file is updated. Figure 2. shows an overview of the DSSMC architecture. 


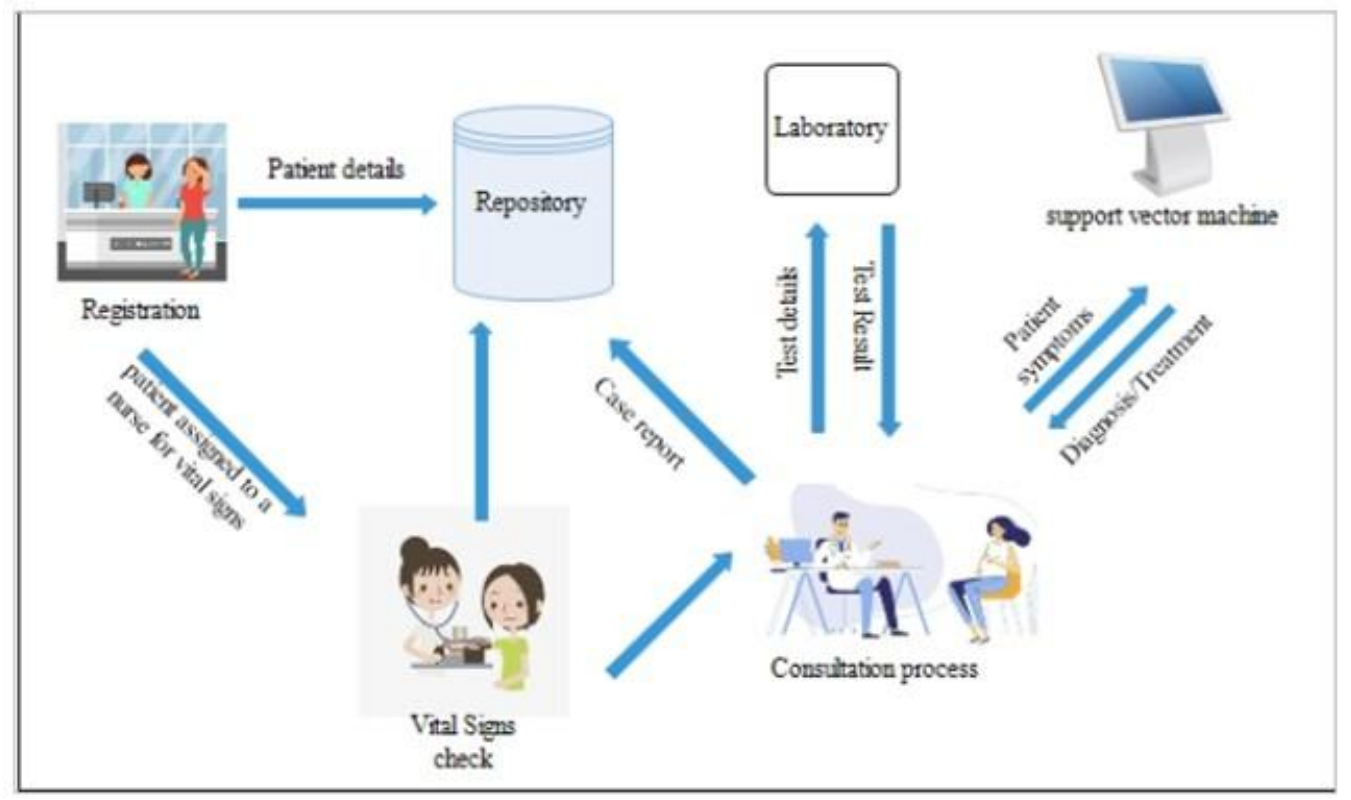

Fig 2: Architecture of Decision Support System for Maternity Health Care (DSSMC)

\subsection{Automated Patient Management}

\section{System}

DSSMC provides an interface where patients/users are registered by providing their health records to the system for diagnosis and analysis. Figure 3, 4 and 5 depict the Context Flow Diagram, Use-Case Diagram and Level-0 Data Flow Diagram of DSSMC respectively.

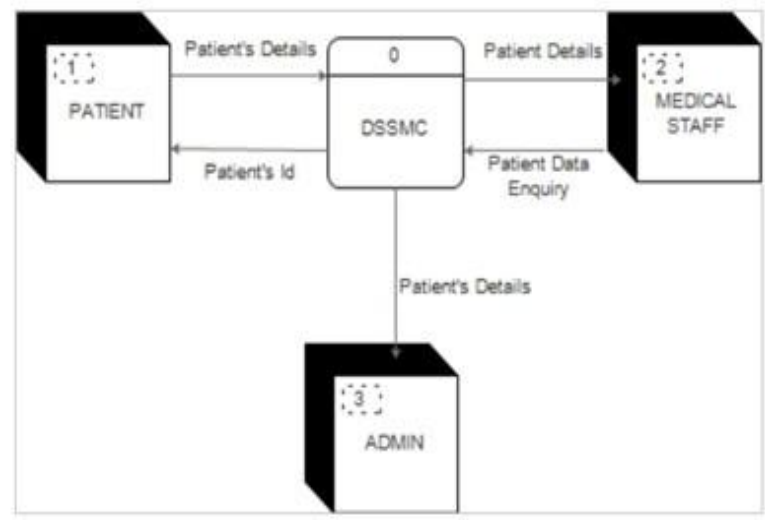

Fig 3: Context Flow diagram for the DSSMC.

In Figure 4, the stages for designing and implementing DSSMC is shown with the use of UML use-case diagram which shows the interaction between the users and the system. There are three (3) users for the DSSMC system which are: the staff, doctor, and admin.

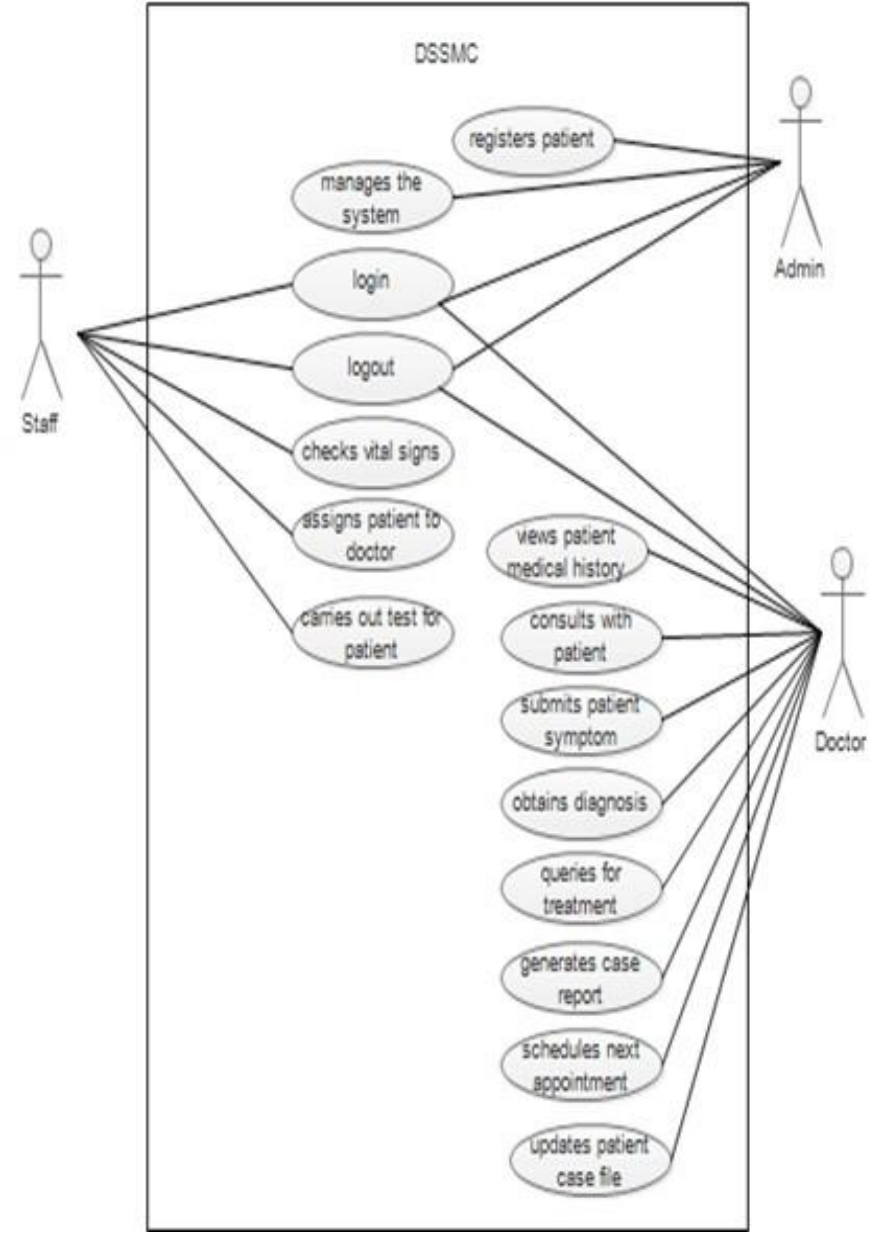

Fig 4: Use-Case Diagram for DSSMC System 


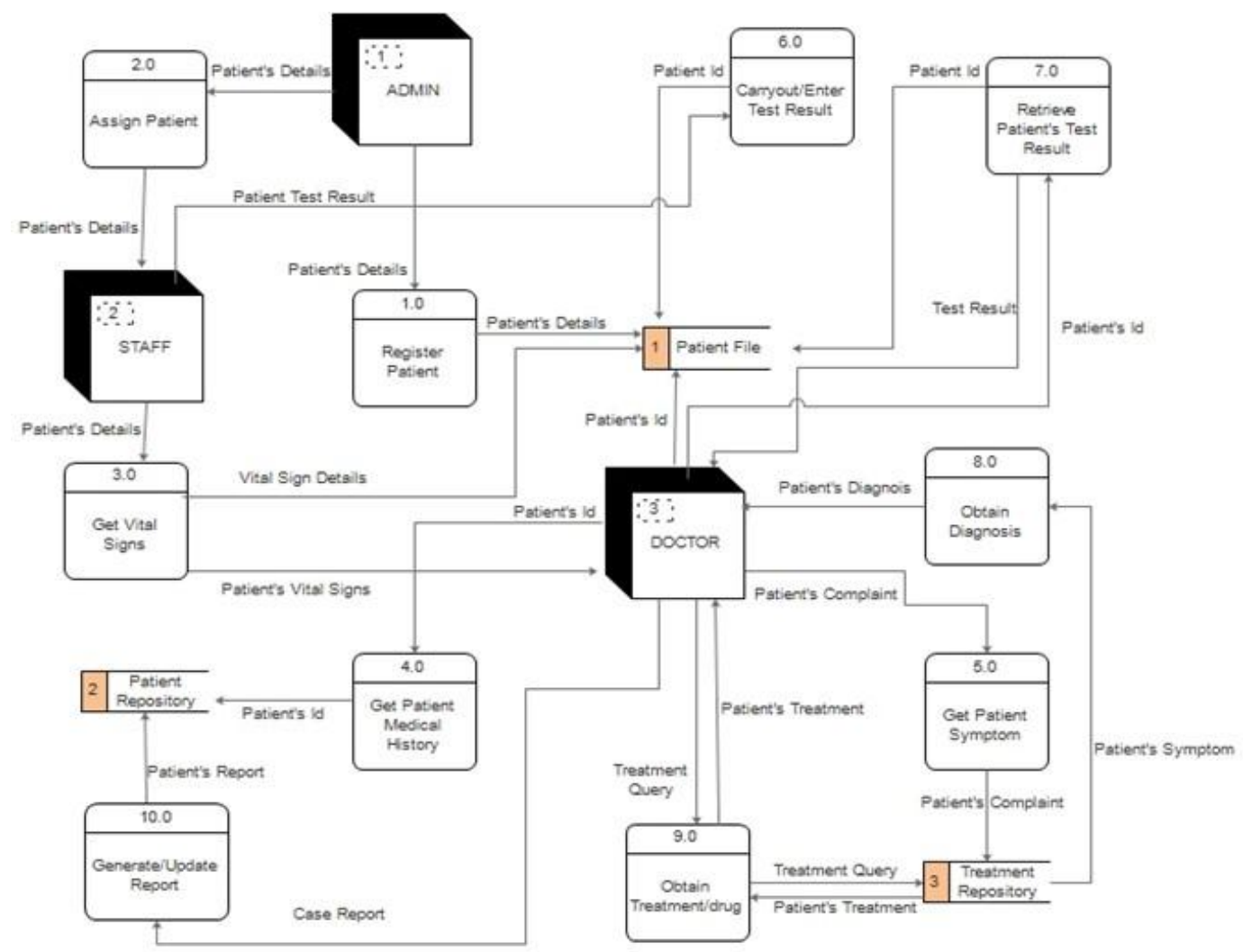

Fig 5: Level-0 Data Flow Diagram of Decision Support System for Maternity Health Care (DSSMC).

\subsection{DSSMC Repository}

All data gathered at the patient registration stage are stored in the repository. The repository acts as a storage medium for the current and historical operational data of patients where it is being pre-processed and validation to serve its decisionmaking objectives. Figure 6 shows the Entity Relationship Diagram of DSSMC.

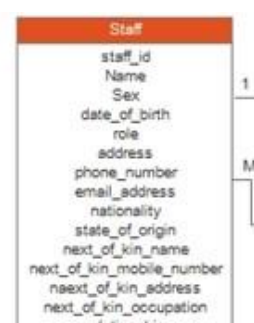

reiasionship

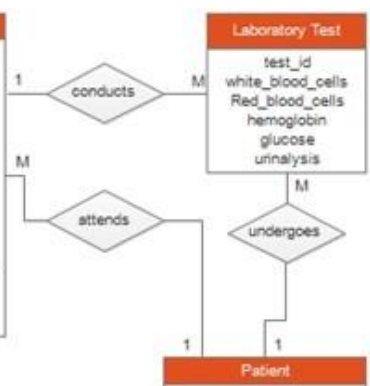

\section{Tet.}
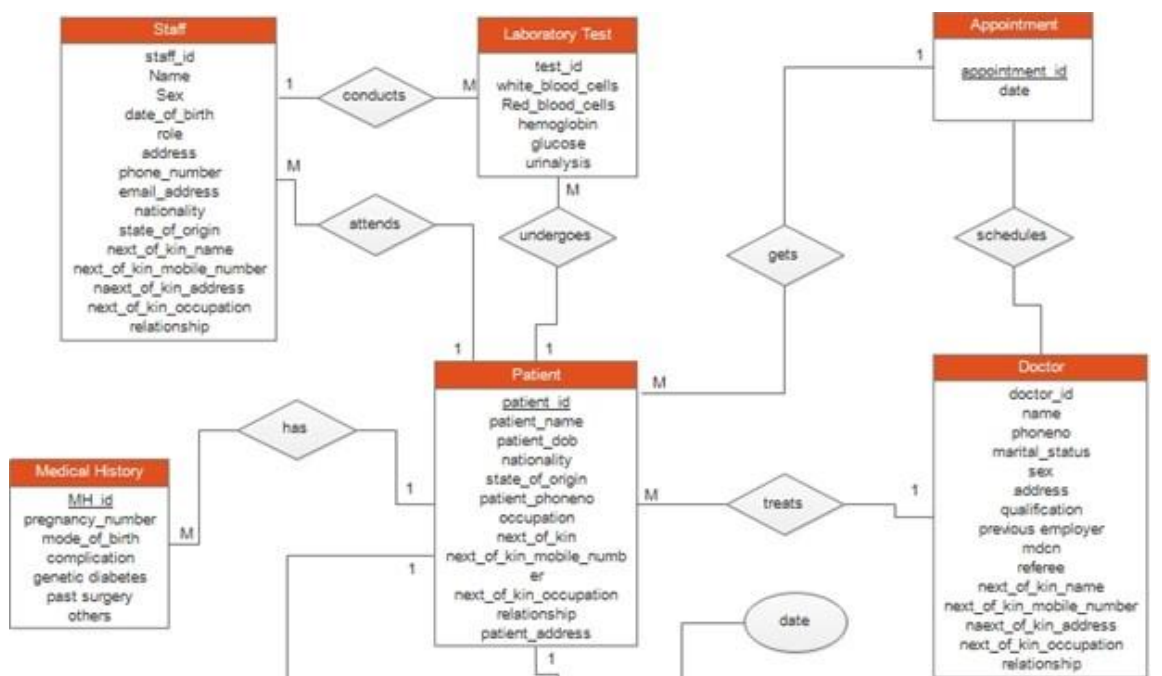
patient_name patient_dob

atorigin occupation of_kin_mobile_num others

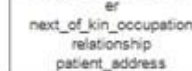

reiationshp
patent_adoress
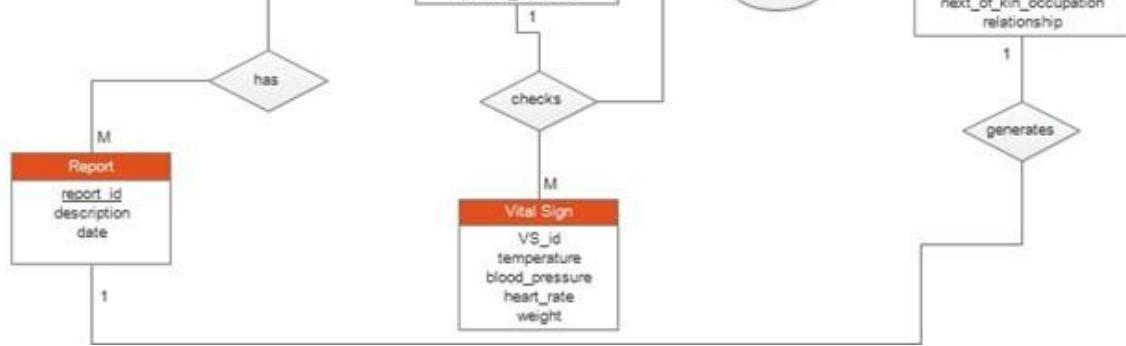

Fig 6: Entity Relationship Diagram for DSSMC 


\subsection{Support Vector Machine (SVM)}

To perform prediction on patients records, a machine learning algorithm called Support Vector Machine (SVM) is used, which inputs patient case file that contains the patient vital signs, laboratory test and medical history that has been captured in the system. It is examined for salient features that will serve as the training dataset for the prediction model that would be trained. The salient features are further examined for its importance in the overall performance of the prediction model. The processed data is used to train a multi-class classification algorithm based on SVM. The main output of this system would be a prediction of patient diagnosis and treatment. The code snippet below shows the pseudo-code for the SVM algorithm. Figure 7 below shows the pseudo-code of the SVM algorithm used in the DSSMC system.

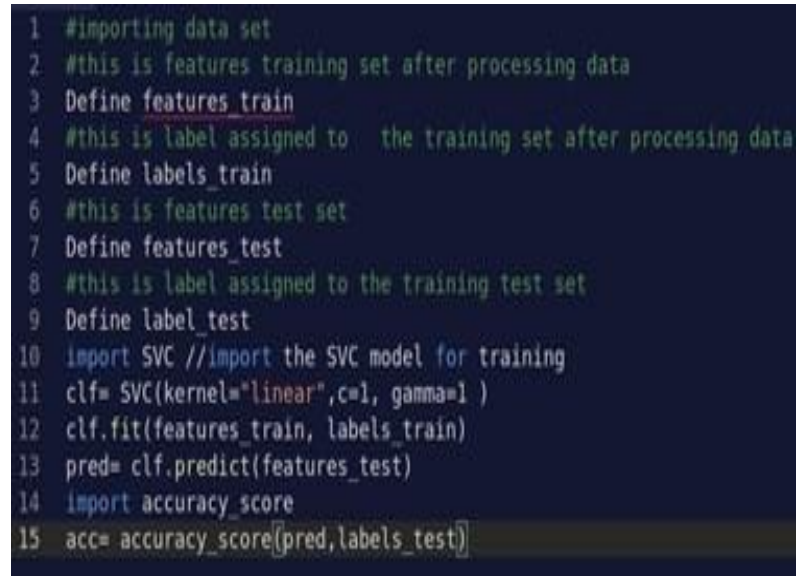

Fig 7: Support Vector Machine (SVM) Algorithm for DSSMC

In figure 7 above, line 2-5 imports the training set and test that will be used to train and evaluate the model i.e. the dataset for the model. Line 6 imports the support vector classifier (SVC) while Line 7 specifies the kernel, the 'c' value for crossevaluation and the gamma value. The kernel is mainly used to find the similarity in the datasets, in this Support vector classifier, it defines the linear hyperplane when training to the algorithm, the output comes out as a classifier. Line 8 passes training data to the fit function where the linear kernel is used as the hyperplane. Line 9 passes the features test for prediction. Line 10 imports accuracy_score which will be used to determine the accuracy of prediction made in line 9. In line 11, the accuracy_score takes in the prediction of line 9 and the label_test to determine the accuracy level of the algorithm.

\section{SYSTEM TESTING OF DSSMC}

Maternity health care provision has exceeded thinking so deep to give drug prescriptions and diagnose a patient, with the use of the DSSMC, diagnoses and treatment are predicted automatically by the system just by clicking a button using a mouse or keyboard. Delayed decision making can lead to inefficient health care provision which can put the lives of patients in danger. The problem of the existing system led to the development and implementation of a decision support system for faster, accurate and efficient decision making. The DSSMC was modelled using use-case for the interactive modelling, data flow diagram was used to model the system processes and an entity-relationship diagram was used to show that the system was implemented in relationship of the system. A web-based decision support system was developed, and the software technologies used are Javascript, node.js and python. A supervised machine learning algorithm based on classification method with support vector machine was used for the prediction model. The software scope was defined and programmed to meet defined specifications. Figure 8 shows the patients registration page of the system. The patient case file which contains the patient vital signs, laboratory test and medical history that has been captured in the system enables prediction of diagnoses and treatment to be done. To get a prediction, the diagnoses button is clicked by the doctor, which displays the predicted diagnoses as shown in figure 9 then a corresponding treatment is predicted for the diagnoses as shown in figure 10 and 11 respectively.

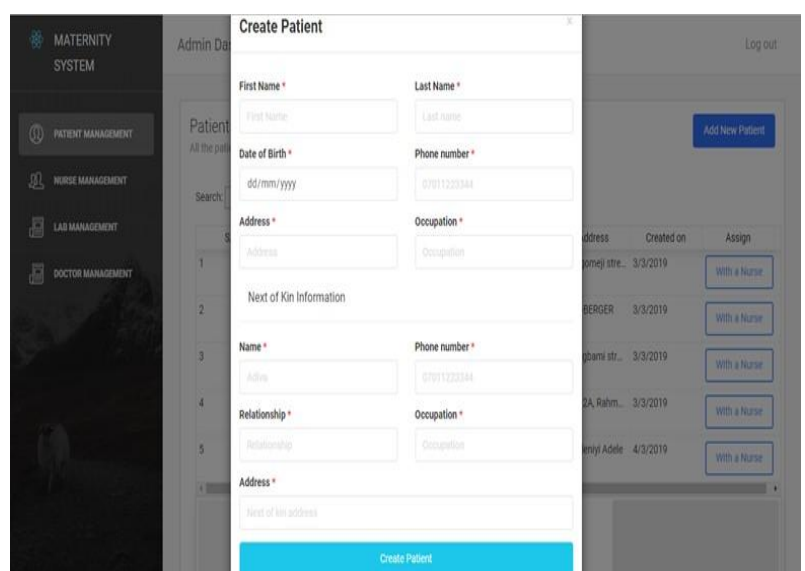

Fig 8: Patient Registration Form for DSSMC System

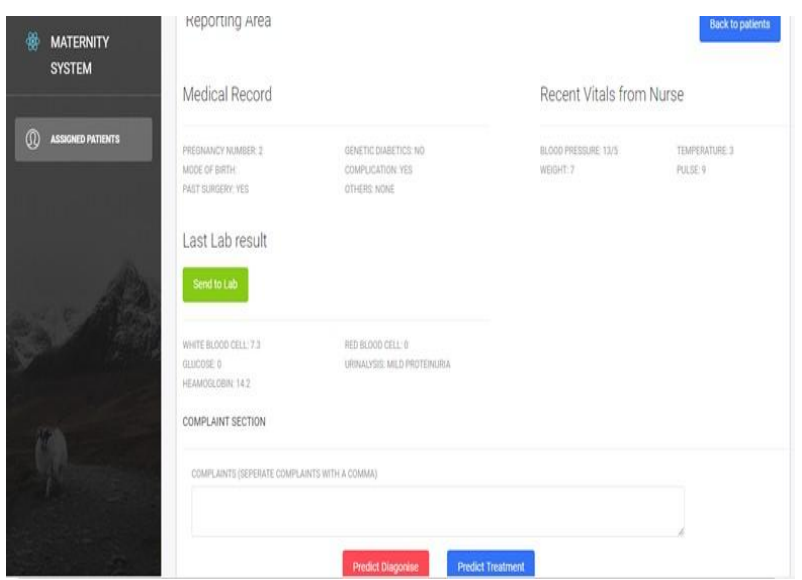

Fig 9: Reporting area of DSSMC

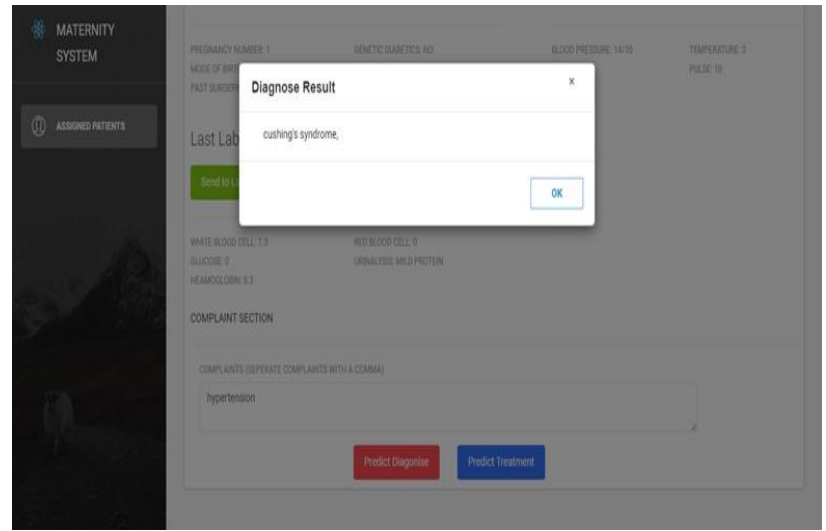

Fig 10: Diagnoses Prediction Result of DSSMC 


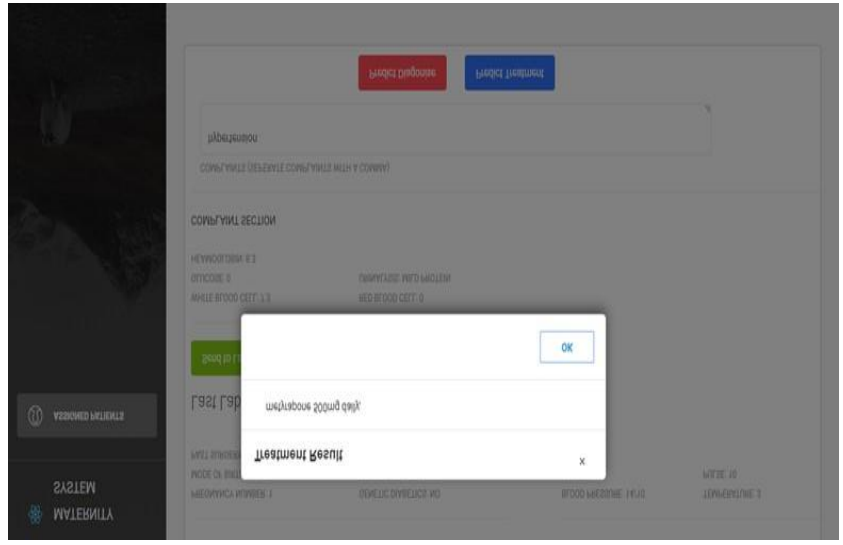

Fig 11: Treatment Result of DSSMC

\section{CONCLUSION AND RECOMMENDATION}

To reduce complications and death in maternity patients, it will be of utmost importance to make timely decisions by health care providers. The development and implementation of a decision support system for maternity health care to enable health care practitioners in making timely and efficient decisions will reduce the rate of maternal complications and death in the society. This research provides a system that offers a decision support functionality to the health care providers, which will enable easy access of patient information, and helps in predicting diagnoses and treatment to specified symptoms, by so doing, the rate of maternal complications will reduce. The DSSMC was developed to enable health care practitioners to make timely and efficient decisions rather than having to think about the likely diagnoses of a particular symptom, as well as the corresponding treatment to give. Further works could include the integration of chatbot for collaboration withing medical practitioners.

\section{ACKNOWLEDGMENTS}

The authors would like to appreciate Ms Kafila Ogundare for her immense contributions towards the success of this study.

\section{REFERENCES}

[1] Abderrazak S., Amina N., Abdelkamel T., Ramtani T., Ouhab A.(2017). Decision Support Health Care Resources Allocation. System for

[2] Addai I. Demographic and sociocultural factors influencing use of maternalhealth services in Ghana. Afr J Reprod Health. 1998;2(1):73-80.17.

[3] Agrawal A. (2002). Return on investment analysis for a computer-based patient record in the outpatient clinic setting. Journal of the Association for Academic Minority Physicians: the official publication of the Association for Academic Minority Physicians, 13(3), 61-65.

[4] Chaudy Yaelle, Connolly Thomas, Magowan Brian \& Soflano Mario. (2017). SAFER Maternity: A Clinical Decision Support System with an Authoring Tool for Clinicians.

[5] Christo El Morr, Julien Subercaze(2010), knowledge management in healthcare. Yorku Components of information systems, https://www.britannica.com/list/5components-of-information-systems(accessed Dec, 2018).
[6] Garg AX, Adhikari NKJ, McDonald H, et al. Effects of Computerized Clinical Decision Support Systems on Practitioner Performance and Patient Outcomes: A Systematic Review. JAMA. 2005;293(10):1223-1238. doi:10.1001/jama.293.10.1223

[7] Karel Fuka, Elina Syrjanen, Rudolf Hanka. (2019). Knowledge Management in Healthcare.Researchagate.

[8] Kathrin C., Azeem M., David W.B., AAziz S. (2012). Computerised Decision Support Systems for HealthCare Professionals: An interpretative Review.researchgate.

[9] Kawamoto, Kensaku \& Houlihan, Caitlin \& Balas, Andrew \& Lobach, David. (2005). Improving Clinical Practice Using Clinical Decision Support Systems: A Systematic Review of Trials to Identify Features Critical to Success. BMJ (Clinical research ed.). 330. 765. 10.1136/bmj.38398.500764.8F.

[10] Leila Shahmoradi., Reza safadari., Worku Jimma(2017), knowledge management implememtation and the tools utilized in healthcare for evidence-based decision making: A systematic Review. Ncbi.

[11] Menachemi N. and Brooks R. G. (2006). Reviewing the benefits and costs of electronic health records and associated patient safety technologies. Journal of Medical Systems, 30(3), 159-168.

[12] Nor;Ashikin Ali, Alexei Tretiakov, Dick Whiddett, Ingaa Hunter (2017), knowledge management systems success in healthcare:Leadership matters.sciencedirect.

[13] O.A. Bolarinwa, A.G. Salaudeen, T.M. Akande(2012), overview of knowledge management applications in health care delivery of developing countries.savap.

[14] Okolocha, C.; Chiwuzie, J; Braimoh, S; Unuigbe, J. and Olumeko, P. (1998). "Socio-cultural factors in Maternal Morbidity and Mortality: A Study of A Semi-Urban Community in Southern Nigeria". Journal of Epidemiology and Community Health, 52(5): 293 - 297.

[15] Paydar, Khadijeh \& Rostam Niakan Kalhori, Sharareh \& Akbarian, Mahmoud \& Sheikhtaheri, Abbas. (2016). A clinical decision support system for prediction of pregnancy outcome in pregnant women with systemic lupus erythematosus. International Journal of Medical Informatics. 97. 10.1016/j.ijmedinf.2016.10.018.

[16] Population Reference Bureau. (2002). Making Motherhood Safer: Overcoming Obstacles on the Pathway to Care. Washington: Population Reference Bureau.

[17] Salter, C., Johnston, H.B., and Hengen, N. (1997). Care for post abortion Complications: Saving Women's lives. Population Reports. Series L, No. 10. Baltimore: JohnsHopkinsSchool of Public Health. Vol. Xxv (1).

[18] Seenivasan P., Sujatha S., Caroline P. K., Saurav D., Srinivasan R., Satya S. P. and Gurlivleen S. G. (2014). Medical Decision Support Technology for Better Antenatal Care of the Mother, Under-Five Child Survival and Child Health in Rural India.

[19] Stroetmann, B., \& Aisenbrey, A. (2012). Medical Knowledge Management in Healthcare Industry.

[20] Van J.B and Musen M.A (1997). Handbook of medical informatics. 
[21] Vinay R. Rao (2018). How data becomes knowledge, part 1. IBM.

[22] Wall, L.L. (1998). Dead Mothers and Injured Wives: The Social Context of Maternal Morbidity and Mortality among the Hausa of Northern Nigeria. Studies in Family Planning, 29 (4).

[23] Wagholikar, Kavishwar \& Maclaughlin, Kathy \& Kastner, Thomas \& Casey, Petra \& Henry, Michael \& Greenes, Robert \& Liu, Hongfang \& Chaudhry, Rajeev. (2013). Formative evaluation of the accuracy of a clinical decision support system for cervical cancer screening.
Journal of the American Medical Informatics Association : JAMIA. 20. 10.1136/amiajnl-2013-001613.

[24] Weed L.L. (1997), Neew connections between medical knowledge and patient care, British medical journal, No. 315, p.231-235.

[25] WHO. (2000). Safe Motherhood: A Newsletter of World Wide Activity. Issue 28(1).

[26] Yaser Hasan Al-mamary, Alina Shamsuddin \& Nor Aziati (2014), the Role of Different types od information systems in business organizations: A review, International journal of research vol 1, issue-7. 